\title{
Renal-protective effect of nicousamide on hypertensive nephropathy in spontaneously hypertensive rats
}

\author{
SEN ZHANG ${ }^{*}$, YAN LI ${ }^{*}$, HONGYAN LI, XUGUANG ZHENG and XIAOGUANG CHEN \\ State Key Laboratory of Bioactive Substances and Functions of Natural Medicines, \\ Department of Pharmacology, Institute of Materia Medica, Chinese Academy of Medical \\ Sciences and Peking Union Medical College, Beijing 100050, P.R. China
}

Received August 2, 2012; Accepted September 19, 2012

DOI: $10.3892 /$ br.2012.11

\begin{abstract}
Previous studies have demonstrated that a novel coumarin-aspirin derivative, nicousamide, has a significant renal-protective effect on diabetic nephropathy. The present study aimed to investigate the beneficial effect of nicousamide on hypertensive nephropathy, as well as the underlying mechanisms in spontaneously hypertensive rats (SHRs). SHRs were treated orally with saline, nicousamide at 15,30 and $45 \mathrm{mg} / \mathrm{kg}$, and losartan $(10 \mathrm{mg} / \mathrm{kg}$ ) daily for 17 weeks, during which blood pressure (BP) was measured every four weeks. At the end of the 17-week treatment, blood and urine samples were collected for biochemical analysis and kidney tissues were obtained for histopathological and reverse transcription-polymerase chain reaction (RT-PCR) analyses. The concentration of angiotensin (Ang) II in plasma was also examined. Results showed that nicousamide effectively attenuated the progression of hypertensive nephropathy by decreasing urinary albumin excretion (UAE) and blood urea nitrogen (BUN). This could significantly decrease BP (less effectively compared to losartan) and the incidence of glomerulosclerosis and glomerular arteriosclerosis, adequately alleviating tubular impairment. Nicousamide markedly reduced the plasma Ang II level in SHRs and reduced mRNA expression of angiotensin-converting enzyme (ACE) and chymase in the kidneys of SHRs. Thus, nicousamide may retard the progression of hypertensive nephropathy. Although the underlying mechanisms have yet to be fully elucidated, this may involve blocking of the renin-Ang system.
\end{abstract}

Correspondence to: Professor Xiaoguang Chen, State Key Laboratory of Bioactive Substances and Functions of Natural Medicines, Department of Pharmacology, Institute of Materia Medica, Chinese Academy of Medical Sciences and Peking Union Medical College, Nan Wei Street 2, Xicheng, Beijing 100050, P.R. China

E-mail: chxg@imm.ac.cn

${ }^{*}$ Contributed equally

Key words: hypertension, nicousamide, nephropathy, angiotensin II, angiotensin-converting enzyme

\section{Introduction}

The renin-angiotensin (Ang) system (RAS) is important in the control of systemic blood pressure (SBP). It is also involved in the pathogenesis of hypertensive renal injury, which is one of the leading causes of chronic kidney disease worldwide $(1,2)$. The analysis of the epidemiological data from dialysis registries has shown that the incidence of end-stage renal disease due to hypertensive nephrosclerosis is progressively increasing in China and a number of other countries. Therefore, novel preventive and therapeutic compounds for hypertensive renal disease should be identified $(3,4)$.

A stage I clinical trial on nicousamide, a potential renal-protective compound, has been completed in China (5), while a stage II clinical trial is to be conducted for the treatment of diabetic nephropathy. Nicousamide is a novel coumarine-aspirin derivative. Recent studies have suggested that nicousamide may reduce the progression of diabetic nephropathy in streptozotocin-induced diabetic rats (6-8). The inhibition of advanced glycation end product (AGE) formation and phosphorylation by transforming growth factor- $\beta$ (TGF- $\beta$ ) receptor II may be associated with the mechanisms underlying the nicousamide-mediated attenuation of diabetic nephropathy, as suggested in previous findings (5). By inhibiting the formation of AGEs, nicousamide blocks the effects of AGEs, such as various intracellular events and increases the activity of various growth factors including TGF- $\beta 1$ and connective tissue growth factor (CTGF) $(9,10)$. By inhibiting the phosphorylation of TGF- $\beta$ receptor II, nicousamide blocks the activity of TGF $\beta$-Smad signal pathways, which are actively involved in the final stage of kidney fibrosis (5).

Spontaneously hypertensive rats (SHRs) have been widely used as a primary hypertension animal model, where the hypertensive nephropathy is characterized by multiple renal structural and functional alterations. Renal injury in SHRs reportedly involves a complex pathological network including renin, angiotensin (Ang) II, monocytes/macrophages, inflammatory cytokines and oxidative stress $(11,12)$. Generally, in SHRs of $>6$ months, the kidney automatically progresses into severe renal injury characterized by marked proteinuria, elevation of serum creatinine ( $\mathrm{Scr}$ ) and blood urea nitrogen (BUN), reduced creatinine clearance ratio $(\mathrm{CCr})$, glomerulosclerosis, interstitial fibrosis and renal vascular arteriosclerosis. These 
characteristics render SHRs a good animal model of human hypertensive nephropathy (13).

Findings of recent studies have shown that the use of RAS inhibitors, such as Ang-converting enzyme inhibitors (ACEs) and Ang receptor blockers (ARBs), may effectively suppress the progression of established renal disease $(14,15)$. These studies suggest the possibility of the early inhibition of RAS as an effective strategy to prevent chronic kidney disease development. In the present study, we aimed to use SHRs to investigate whether or not nicousamide could alleviate chronic kidney injury under hypertensive conditions.

\section{Materials and methods}

Ethical considerations. This study was approved by the Institutional Animal Care Committee of the Peking Union Medical College (Beijing, China), and was conducted in accordance with the US National Institute of Health Guide for the Care and Use of Laboratory Animals.

Animal treatment protocol. Male SHRs (age, 16 weeks; weight, 300-340 g) were obtained from the Institute of Laboratory Animal Science, the Chinese Academy of Medical Sciences (Beijing, China). They were kept in a specific pathogen-free facility and provided with adequate food and water ad libitum, under constant temperature $\left(22 \pm 2^{\circ} \mathrm{C}\right)$ conditions, with a $12-\mathrm{h}$ (7:00 a.m.-7:00 p.m.) dark-light cycle.

Rats were randomized into 5 groups $(n=10)$ : i) no treatment control; ii) losartan [10 mg/kg, periocular (p.o.)] treatment; iii) oral nicousamide at $15 \mathrm{mg} / \mathrm{kg}$; iv) oral nicousamide at $30 \mathrm{mg} / \mathrm{kg}$ and $\mathrm{v}$ ) oral nicousamide at $45 \mathrm{mg} / \mathrm{kg}$. Nicousamide with a purity of $99 \%$ was synthesized by the Department of Medicinal Chemistry, Institute of Materia Medica, the Chinese Academy of Medical Sciences (Beijing, China). Losartan and nicousamide were diluted in sodium carboxymethycellulose-Na (CMC-Na). The treatment continued for up to 17 weeks, during which $\mathrm{BP}$ and heart rate (HR) were measured every 4 weeks by tail-cuff plethysmography (BP-98A; Softron, Tokyo, Japan) with prior training to minimize variability in BP measurement.

Ten age-matched normotensive Wistar rats were also raised under the same conditions as the normal controls.

Chemical analyses of blood and urine. At the end of the designated treatments, blood was sampled through the eyes under anesthesia with diethyl ether. Urine samples were continuously collected for $24 \mathrm{~h}$ from each animal after placement in metabolic cage, the day prior to blood sample collection. BUN and urinary albumin excretion (UAE) were measured using the standard biochemical kits (Beijing BHKT Clinical Reagent Co., Ltd., Beijing, China), respectively. $\mathrm{CCr}$ was calculated according to the formula: $\mathrm{CCr}=$ urinary creatinine $(\mathrm{mg} / \mathrm{ml}) \times$ urine volume $(\mathrm{ml} / \mathrm{kg}) /$ creatinine in plasma $(\mathrm{mg} / \mathrm{ml})$.

Histopathological assessment. At the end of the 17-week treatment, the rats were anaesthetized by ether and sacrificed. Kidneys and hearts were removed and weighed. The weight ratio of the kidney or heart to the body was calculated and termed kidney or heart index. Tissue blocks of kidneys were fixed in $10 \%$ formalin solution, embedded in paraffin and sectioned. The sections were stained with Periodic Acid-Schiff (PAS) and haematoxylin and eosin (H\&E) to evaluate glomerulosclerosis. The percentage of sclerosis-positive glomeruli was calculated. At least 30 glomeruli localized within a 1-mm depth from the surface of the kidney cortex were analyzed in each section. Interstitium-tubular injuries were classified into four categories: interstitium infiltration, interstitium fibrosis, tubular dilatation and tubule-interstitium protein casts. The incidence in each group was determined semi-quantitatively.

Determination of Ang II level in plasma. Ang II level in plasma was determined using the Angiotensin II ELISA kit (Enzo Biochem Inc., New York, NY, USA), according to the detailed procedure described in the manufacturer's manual.

Reverse transcription-polymerase chain reaction (RT-PCR). In order to further investigate the mechanism underlying the effect of nicousamide on the RAS system, mRNA levels of renin, ACE and chymase in kidney tissues were semi-quantitated by RT-PCR.

Total RNA was extracted from the fresh kidney tissues of animals using TRIzol reagent. For each RNA sample, $2 \mu \mathrm{g}$ RNA were reverse-transcribed into cDNA using oligo(dT) random primers, with the SuperScript ${ }^{\mathrm{TM}}$ II RNase H Reverse Transcriptase. PCR was performed with reaction mixtures containing $2.5 \mathrm{mM}$ dNTP, $10 \mathrm{mM}$ sense and antisense primers, and $5 \mathrm{U} / \mathrm{ml}$ TaqDNA polymerase in a thermal cycler for $30 \mathrm{sec}$ at $94^{\circ} \mathrm{C}, 30 \mathrm{sec}$ at $58^{\circ} \mathrm{C}$ ( $\beta$-actin), $58^{\circ} \mathrm{C}$ (renin), $65^{\circ} \mathrm{C}(\mathrm{ACE})$, $59^{\circ} \mathrm{C}$ (chymase) and $1 \mathrm{~min}$ at $72^{\circ} \mathrm{C}, 35$ cycles. The final extension reaction was performed at $72^{\circ} \mathrm{C}$ for $5 \mathrm{~min}$.

The PCR primers used in this study were: renin, sense: 5'-GTGCAGCCGTCTCTAC-3' and antisense: 5'-CCG TGACCTCTCCAAAC-3'; ACE, sense: 5'-GCAAGGAGG CAGGCTATGAG-3' and antisense: 5'-CGGGTAAAACT GGAGGATG G-3'; chymase, sense: 5'-CTGAGAGGATGC TTCTTCCTGC-3' and antisense: 5'-AGATCTTATTGATCCA GGGCCG-3'; $\beta$-actin was used as an internal control, sense: 5'-GTGGGGCGCCCCAGGCACCA-3' and antisense: 5'-CTTCCTTAATGTCACGCACGATTTC-3'. The PCR products were electrophoresed on $1.5 \%$ agarose gels, visualized by ethidium bromide staining, and photographed under ultraviolet (UV) light. PCR was repeated three times and the average optical density was analyzed using the ImageJ software.

Statistical analysis. Data were expressed as the mean \pm standard deviation (SD). Statistical comparisons were performed using the Student's t-test, with the exception of the histological analyses in interstitium-tubular lesions, which were analyzed using the Pearson's Chi-square test. $\mathrm{P}<0.05$ was considered to indicate a statistically significant difference.

\section{Results}

Effects of nicousamide on BP and HR. Prior to drug treatment, the average systolic arterial pressure (SAP) of SHRs was $200 \mathrm{mmHg}, 56 \mathrm{mmHg}$ higher compared to normotensive rats $(\mathrm{P}<0.01$, Fig. 1A). The average diastolic pressure (DAP) was $172 \mathrm{mmHg}, 50 \mathrm{mmHg}$ higher compared to normotensive rats 
A

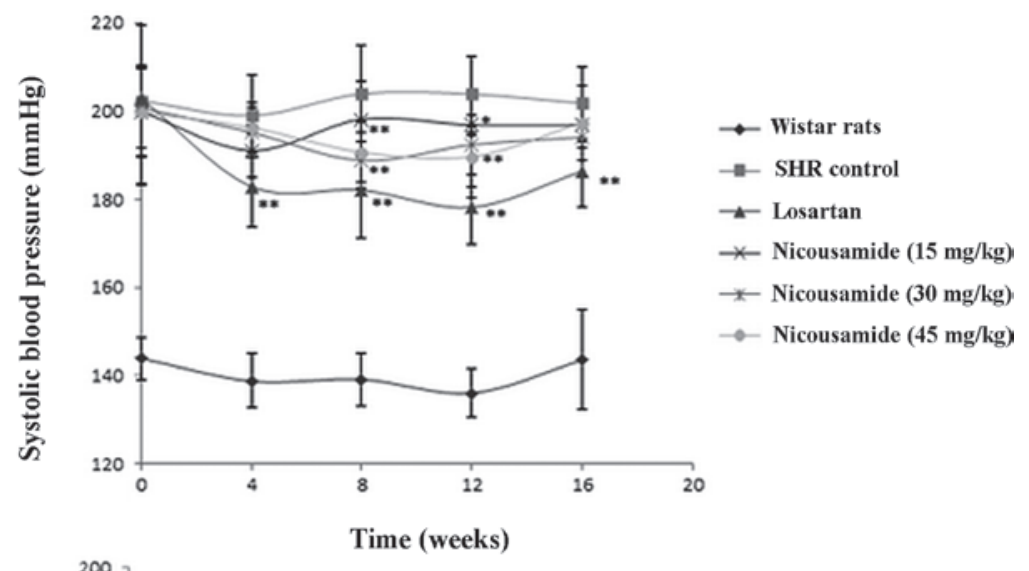

B

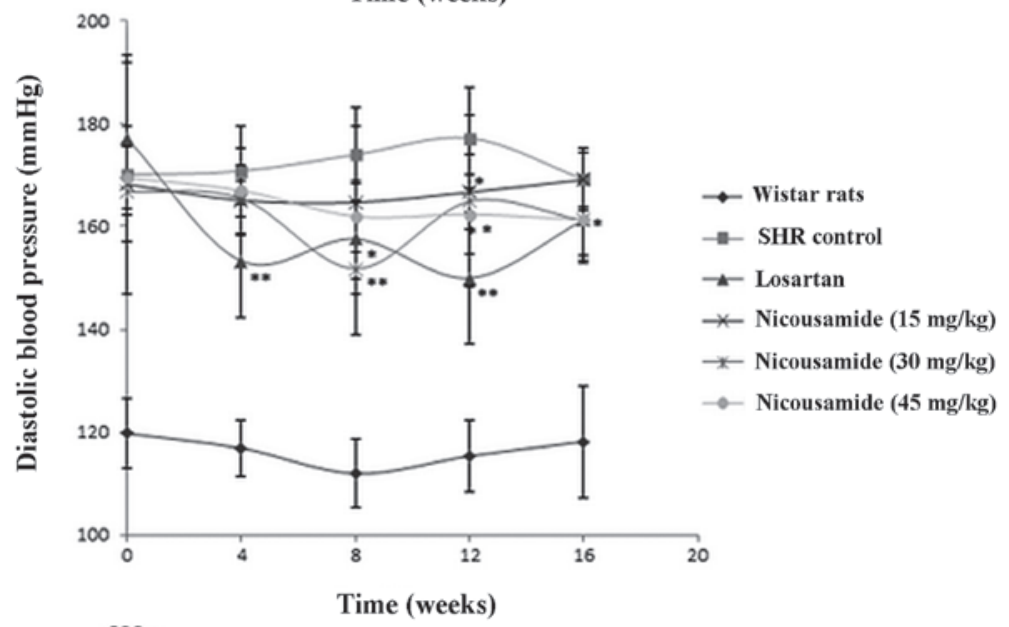

C

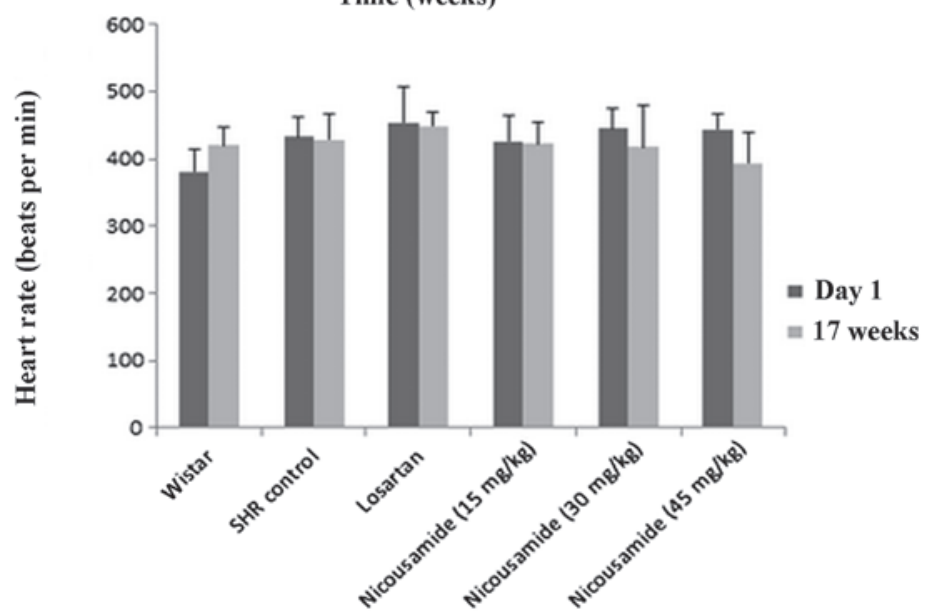

Figure 1. Effects of nicousamide and losartan on (A) average systolic pressure (SAP), (B) average diastolic pressure (DAP) and (C) heart rate (HR) in SHRs after a 17-week treatment are shown. Nicousamide markedly decreased SAP and DAP at doses of $30 \mathrm{and} 45 \mathrm{mg} / \mathrm{kg}$, although its effect was weaker compared to losartan $(10 \mathrm{mg} / \mathrm{kg}) .{ }^{*} \mathrm{P}<0.05 ;{ }^{* *} \mathrm{P}<0.01$ when compared to untreated control SHRs group. SHRs, spontaneously hypertensive rats.

( $\mathrm{P}<0.01$, Fig. 1B). The HR of SHRs was also higher compared to normotensive rats $(\mathrm{P}<0.05)$ (Fig. $1 \mathrm{C})$.

Subsequent to drug treatment, BP was measured every four weeks and the results are shown in Fig. 1A and B. Compared to untreated SHRs, SHRs in the nicousamide treatment groups showed a decreased SAP and DAP, especially at doses of 30 and $45 \mathrm{mg} / \mathrm{kg}(\mathrm{P}<0.05$ or $\mathrm{P}<0.01)$, however, its BP-lowering effect was less effective compared to losartan.

No statistically significant difference was observed on HRs among the groups of experimental SHRs after the 17 -week treatment, although nicousamide $(45 \mathrm{mg} / \mathrm{kg})$ treat- ment decreased HR by $8.24 \%$ compared to untreated SHRs, no statistically significant difference was found (Fig. 1C).

Benefits of nicousamide on renal function. The results of BUN, CCr and UAE measurements are presented in Table I. Compared to normotensive rats, untreated SHRs showed markedly higher levels in these three parameters $(\mathrm{P}<0.01)$.

Treatment with nicousamide at $15 \mathrm{mg} / \mathrm{kg}$ decreased BUN level by $16.01 \%(\mathrm{P}<0.05)$ as compared to the untreated control $(\mathrm{P}<0.05)$. Treatment of SHRs with nicousamide at $45 \mathrm{mg} / \mathrm{kg}$ improved $\mathrm{CCr}$ values by $16.73 \%$ as compared to the control 
Table I. UAE, BUN, and CCr values after a 16-week nicousamide treatment in SHRs.

\begin{tabular}{|c|c|c|c|c|c|c|}
\hline \multirow[b]{2}{*}{ Parameters } & \multirow{2}{*}{$\begin{array}{l}\text { Normotensive } \\
\text { control }\end{array}$} & \multirow{2}{*}{$\begin{array}{l}\text { Control } \\
\text { SHRs }\end{array}$} & \multirow{2}{*}{$\begin{array}{l}\text { Losartan } \\
(10 \mathrm{mg} / \mathrm{kg})\end{array}$} & \multicolumn{3}{|c|}{ Nicousamide (mg/kg) } \\
\hline & & & & 15 & 30 & 45 \\
\hline No. & 10 & 10 & 10 & 10 & 10 & 10 \\
\hline $\mathrm{UAE}(\mathrm{mg} / \mathrm{ml} /$ day $)$ & $21.47 \pm 6.91$ & $24.76 \pm 8.23^{\mathrm{a}}$ & $19.11 \pm 5.86^{\mathrm{b}}$ & $20.08 \pm 10.04^{\mathrm{b}}$ & $25.32 \pm 6.46$ & $24.58 \pm 4.50$ \\
\hline BUN (mg/dl) & $17.79 \pm 2.06$ & $23.88 \pm 1.78^{\mathrm{a}}$ & $21.76 \pm 2.12^{\mathrm{b}}$ & $20.06 \pm 0.95^{\mathrm{b}}$ & $23.84 \pm 2.22$ & $22.82 \pm 2.33$ \\
\hline $\mathrm{CCr}(\mathrm{ml} / \mathrm{min} / 100 \mathrm{~g}$ body weight $)$ & $1.57 \pm 0.47$ & $0.77 \pm 0.39^{\mathrm{a}}$ & $1.23 \pm 0.33^{\mathrm{b}}$ & $0.83 \pm 0.26$ & $0.80 \pm 0.22$ & $0.83 \pm 0.15$ \\
\hline
\end{tabular}

${ }^{\mathrm{a}} \mathrm{P}<0.01$ vs. normotensive control rats; ${ }^{\mathrm{b}} \mathrm{P}<0.05$ vs. control SHRs. SHRs, spontaneously hypertensive rats.

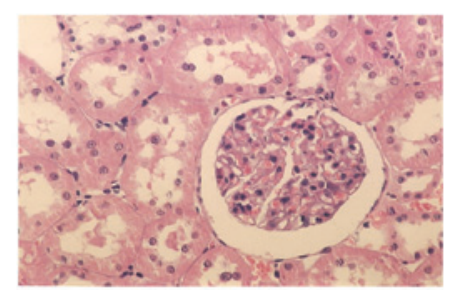

\begin{tabular}{l|l|l} 
Normal (HE, 100X) & & \\
\hline SHR (HE, 100X) & SHR (PAS-M, 100X) & $\begin{array}{l}\text { Renal arteriosclerosis of SHR (HE, } \\
100 X)\end{array}$ \\
\hline $\begin{array}{l}\text { SHR + 45 mg } / \mathrm{kg} \\
\text { nicousamide }(H E, 100 X)\end{array}$ & $\begin{array}{l}\text { SHR }+45 \mathrm{mg} / \mathrm{kg} \text { nicousamide } \\
\text { (PAS-M, 100X) }\end{array}$ & $\begin{array}{l}\text { Renal arteriosclerosis of SHR + } \\
45 \mathrm{mg} / \mathrm{kg} \text { nicousamide }(H E, 100 X)\end{array}$
\end{tabular}
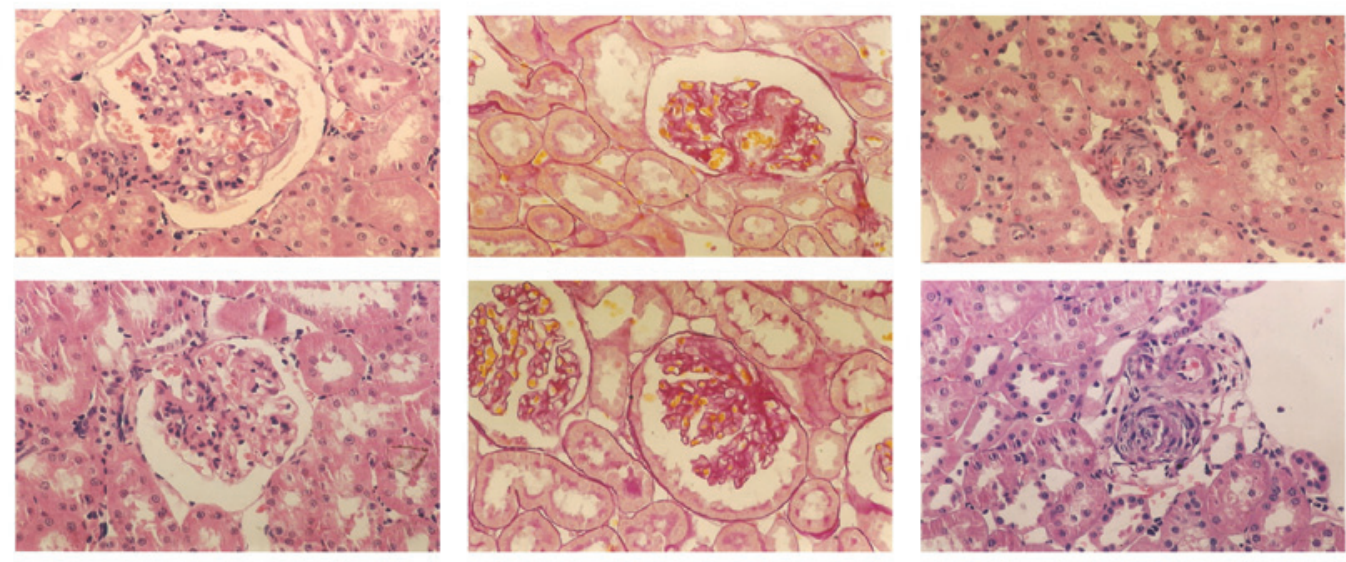

Figure 2. Representative images of the haematoxylin and eosin (H\&E)- and Periodic Acid-Schiff (PAS)-stained kidney tissue sections collected from normotensive rats and SHRs in the control and nicousamide-treated groups are shown.

$(\mathrm{P}<0.05)$. A modest improvement on UAE level was observed at a $15-\mathrm{mg} / \mathrm{kg}$ dose of nicousamide treatment.

Benefits of nicousamide on kidney histopathology. Histopathological findings in SHRs in various treatment groups and in normotensive rats are shown in Fig. 2 and Table II. In the absence of drug treatments, SHRs exhibited higher indices in kidney and heart indices $(\mathrm{P}<0.01)$, glomerulosclerosis and apparent histological abnormalities in the interstitium-tubular areas including interstitium infiltration of inflammatory cells, fibrosis, tubular dilatation and protein casts, as compared to normotensive rats.

Treatment with either nicousamide or losartan for 17 weeks did not markedly improve the kidney or heart index, although a modest amelioration was observed $(\mathrm{P}>0.05)$. By contrast, nicousamide treatment resulted in significant alleviation of glomerulosclerosis $(\mathrm{P}<0.05)$. When used at 15 and $30 \mathrm{mg} / \mathrm{kg}$, amelioration of glomeruloscerosis was evident $(\mathrm{P}<0.05)$, while when used at $45 \mathrm{mg} / \mathrm{kg}$, the level of glomerulosclerosis decreased from 12.34 to $4.66 \%(\mathrm{P}<0.05)$. At the three doses, nicousamide markedly reduced interstitium infiltration of inflammatory cells, fibrosis, tubular dilatation and protein casts in SHRs $(\mathrm{P}<0.05)$. In the present study, nicousamide showed a stronger effect compared to losartan on histopathological improvements.

Nicousamide decreased Ang II concentrations of plasma in SHRs. As shown in Fig. 3, the Ang II level in the nicousamide-treated groups was reduced in a dose-dependent manner compared to the untreated controls. At the dose of $45 \mathrm{mg} / \mathrm{kg}$, nicousamide reduced the plasma Ang II by $27.2 \%$ $(\mathrm{P}<0.05)$, while losartan markedly increased plasma Ang II level $(\mathrm{P}<0.01)$.

Effects of nicousamide on renin, ACE and chymase $m R N A$ levels. As shown in Fig. 4, SHRs demonstrated higher mRNA levels of chymase and ACE in kidney tissues compared to normotensive rats. Using optical density analysis, we found that nicousamide and losartan could slightly but markedly decrease ACE and chymase mRNA levels in SHR kidney tissues, especially at $45 \mathrm{mg} / \mathrm{kg}(\mathrm{P}<0.05)$. Although nicousamide also 
Table II. Kidney histopathological characteristics of the rats at the17th week.

\begin{tabular}{lcccccc}
\hline & & & & \multicolumn{2}{c}{ Nicousamide (mg/kg) } \\
\cline { 5 - 7 } Parameters & $\begin{array}{c}\text { Normotensive } \\
\text { control }\end{array}$ & $\begin{array}{c}\text { Control } \\
\text { SHRs }\end{array}$ & $\begin{array}{c}\text { Losartan } \\
(10 \mathrm{mg} / \mathrm{kg})\end{array}$ & 15 & 30 & 45 \\
\hline No. & 10 & 10 & 10 & 10 & 10 & 10 \\
Kidney index (mg/kg BW) & $0.574 \pm 0.05$ & $0.687 \pm 0.04^{\mathrm{a}}$ & $0.679 \pm 0.03$ & $0.674 \pm 0.05$ & $0.670 \pm 0.07$ & $0.672 \pm 0.02$ \\
Heart index (mg/kg BW) & $0.255 \pm 0.023$ & $0.388 \pm 0.019^{\mathrm{a}}$ & $0.355 \pm 0.016$ & $0.369 \pm 0.012$ & $0.358 \pm 0.026$ & $0.371 \pm 0.018$ \\
Glomerulosclerosis (\%) & $3.67 \pm 4.29$ & $12.34 \pm 4.45^{\mathrm{a}}$ & $6.68 \pm 5.00^{\mathrm{b}}$ & $7.51 \pm 2.52^{\mathrm{b}}$ & $7.32 \pm 4.39^{\mathrm{b}}$ & $4.66 \pm 3.59^{\mathrm{b}}$ \\
Incidence of interstitium infiltration & $10(0)$ & $10(4)^{\mathrm{a}}$ & $10(5)$ & $10(4)$ & $10(4)$ & $10(3)$ \\
Incidence of interstitium fibrosis & $10(0)$ & $10(2)^{\mathrm{a}}$ & $10(2)$ & $10(1)$ & $10(3)$ & $10(0)^{\mathrm{b}}$ \\
Incidence of tubular dilatation & $10(0)$ & $10(4)^{\mathrm{a}}$ & $10(1)$ & $10(1)^{\mathrm{b}}$ & $10(2)$ & $10(1)^{\mathrm{b}}$ \\
Incidence of tubule-interstitium & $10(2)$ & $10(4)^{\mathrm{a}}$ & $10(1)^{\mathrm{b}}$ & $10(2)$ & $10(3)$ & $10(1)^{\mathrm{b}}$ \\
protein casts & & & & & &
\end{tabular}

Values in brackets indicate the number of sever impairment cases among 10 animals. ${ }^{\mathrm{P}}<0.01$ vs. normotensive control rats; ${ }^{\text {b }}<0.05$ vs. untreated SHRs. SHRs, spontaneously hypertensive rats. BW, body weight.

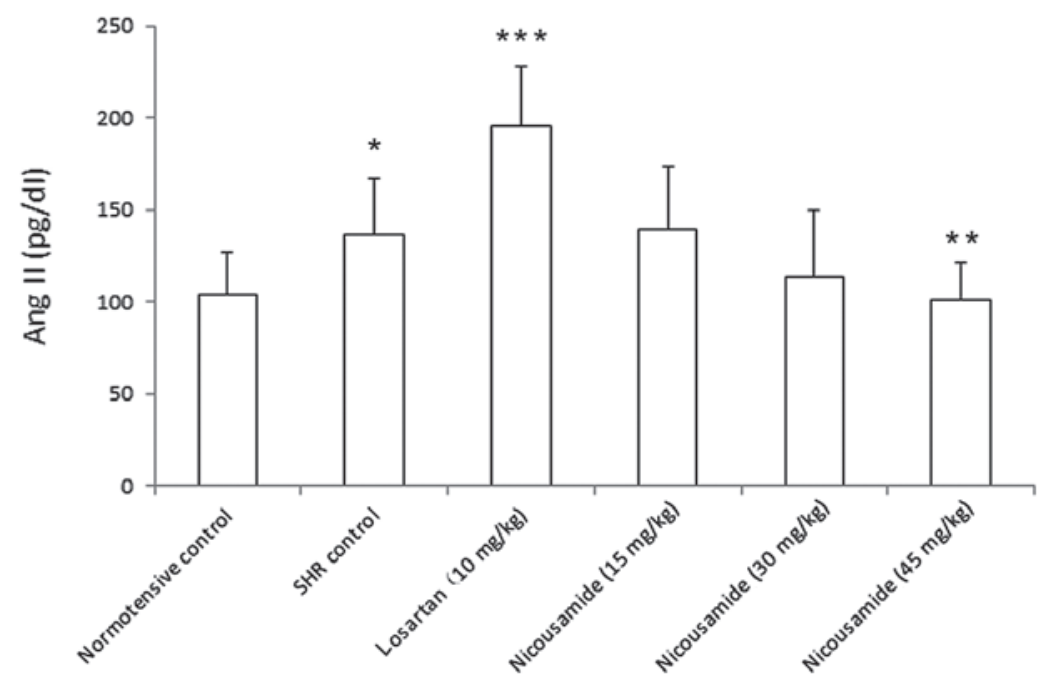

Figure 3. Angiotensin (Ang) II levels in plasma, determined using the ELISA kit, are shown. ${ }^{*} \mathrm{P}<0.05$ vs. normotensive rats, ${ }^{* *} \mathrm{P}<0.05$ and ${ }^{* * *} \mathrm{P}<0.01 \mathrm{vs}$. untreated control SHRs.

showed a certain inhibitory effect on renin mRNA expression in kidney tissues, no statistical significance was observed $(\mathrm{P}>0.05)$.

\section{Discussion}

Nicousamide is a class 1.1 drug protected by domestic and international patents. A phase II clinical trial for diabetic nephropathy is currently being conducted in the Peking Union Hospital (Beijing, China). In addition to its potential in treating diabetic nephropathy, in the present study we aimed to use SHRs as model animals to investigate whether or not it also has a renal-protective effect on hypertensive nephropathy in rats.

In the present study, 16-week-old SHRs were used. At the end of the 17-week treatment, the rats progressed into the stage of chronic renal dysfunction with low creatinine clearance, high UAE and BUN. Nicousamide markedly attenuated renal dysfunction and improved the three renal function parameters, as demonstrated by the biochemical analysis. Compared to losartan, nicousamide was less effective in improving the biochemical parameters of renal function.

Notably, histopathological analysis showed that nicousamide markedly alleviated injuries in the glomeruli and proximal tubules. The incidence of glomerulosclerosis in the nicousamide-treated SHRs was markedly lower compared to that of the control SHRs, and its beneficial effect was even better compared to that of losartan. The impairment in tubulointerstitial fibrosis and other tubular-interstitium injuries (interstitium infiltration, interstitium fiborosis, tubular dilatation and tubule-interstitium protein casts) were also markedly reduced after nicousamide treatment $(\mathrm{P}<0.05)$. These observations suggest a therapeutic potential of nicousamide for hypertensive nephropathy.

Regarding the underlying mechanisms involved in the renal-protective effect on hypertensive nephropathy, we hypothesized that the BP-lowering action constituted one 

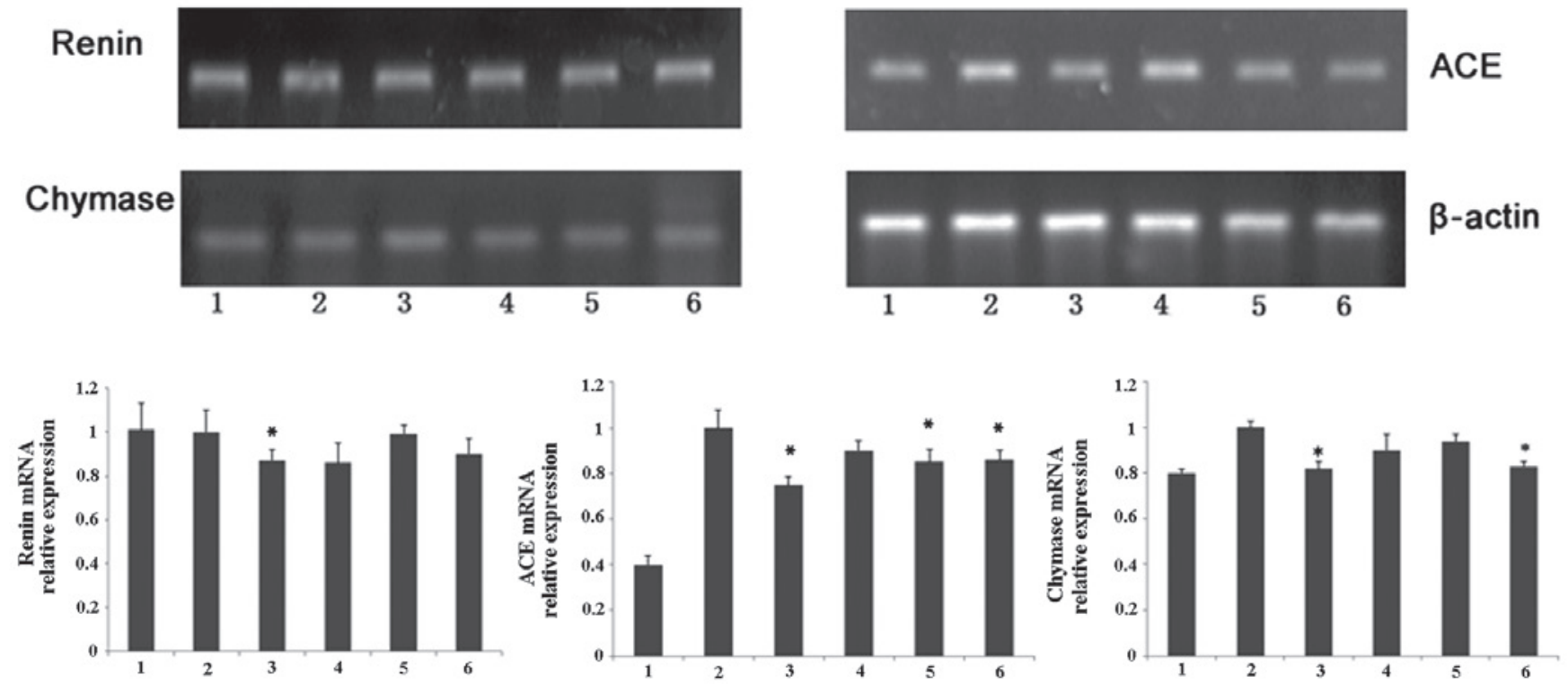

Figure 4. Image of a representative agarose gel of RT-PCR products after ethidium bromide staining is shown. Lanes 1 , Normotensive rats; 2 , untreated control SHRs; 3, SHRs treated with losartan (10 mg/kg); 4, SHRs treated with nicousamide at $15 \mathrm{mg} / \mathrm{kg}$; 5, SHRs treated with nicousamide at 30 mg/kg and 6, SHRs treated with nicousamide at $45 \mathrm{mg} / \mathrm{kg}$. ${ }^{*} \mathrm{P}<0.05$ vs. untreated control SHRs group.

of the most important reasons. Our results have shown that nicousamide markedly decreased SAP and DAP in SHRs during the long-term treatment. Obviously, anti-hypertension is helpful for nicousamide to retard the progression of hypertensive nephropathy.

To investigate the nicousamide effect on the regulation of the BP-lowering action, mRNA levels of certain important components in the RAS system, such as renin, ACE and chymase in the animal kidneys were evaluated using RT-PCR. Renin, produced by collecting duct cells, circulates in the bloodstream and hydrolyzes Ang to Ang I $(16,17)$. In the present study, compared to the control model SHRs, only the losartan-treated group demonstrated a significant decrease in the renin level, and although nicousamide treatment also showed a certain reduction in renin mRNA expression, no statistical significance was found.

Chymase is a serine protease, released by mucosal mast cells upon challenge with parasites and parasite antigens promoting an inflammatory response (18). Chymase is also known to convert Ang I to Ang II and is, thus, crucial in hypertension and atherosclerosis (19). In the present study, losartan and nicousamide $(45 \mathrm{mg} / \mathrm{kg})$ treatment markedly downregulated chymase mRNA expression in the kidneys of SHRs. Thus, it was hypothesized that by inhibiting chymase expression, nicousamide might reduce the concentration of Ang II and finally reduce BP.

$\mathrm{ACE}$, which is present in vascular and tubular epithelium, catalyzes the conversion of decapeptide Ang I to active Ang II (20), potentially inducing strong vascular contraction. In the present study, subsequent to nicousamide treatment (30 or $45 \mathrm{mg} / \mathrm{kg}$ ), the mRNA level of ACE in animal kidneys was slightly but markedly reduced and similar results were also observed in the losartan-treated group. By reducing the ACE expression, nicousamide may decrease the concentration of Ang II in plasma and eventually reduce BP.
Ang II levels in the plasma were assessed in order to confirm our hypothesis. The results, according to which the Ang II level in plasma was reduced in a dose-dependent manner compared to the model group, confirmed our hypothesis. Consequently, decreasing Ang II is crucial in anti-hypertension for nicousamide.

In conclusion, nicousamide may moderately reduce $\mathrm{BP}$, thus having a beneficial effect on individuals with hypertensive nephropathy. This BP-lowering effect may be achieved through the reduction of the plasma Ang II and the decrease of ACE and chymase expression.

\section{Acknowledgements}

The authors appreciate the support provided by the Key Project of the National Eleventh-Five Year Research Program of China.

\section{References}

1. Azegami T, Sasamura H, Hayashi K and Itoh H: Vaccination against the angiotensin type 1 receptor for the prevention of L-NAME-induced nephropathy. Hypertens Res 35: 492-499, 2012.

2. Hill GS: Hypertensive nephrosclerosis. Curr Opin Nephrol Hypertens 17: 266-270, 2008.

3. Chen Y, Lipkowitz MS, Salem RM, et al: Progression of chronic kidney disease: adrenergic genetic influence on glomerular filtration rate decline in hypertensive nephrosclerosis. Am J Nephrol 32: 23-30, 2010.

4. Wang G, Lai FM, Kwan BC, et al: Expression of ACE and ACE2 in patients with hypertensive nephrosclerosis. Kidney Blood Press Res 34: 141-149, 2011.

5. Zhang H, Jin J, Zhou W, et al: Nicousamide, a potent inhibitor of phosphorylation by TGF- $\beta$ receptor II. Acta Pharmaceutica Sinica 1: 160-165, 2011.

6. Li H, Zheng X, Wang H, Zhang Y, Xin H and Chen X: XLF-III-43, a novel coumarin-aspirin compound, prevents diabetic nephropathy in rats via inhibiting advanced glycation end products. Eur J Pharmacol 627: 340-347, 2010. 
7. Li H, Zhang Y, Wang H, Zheng $\mathrm{X}$ and Chen $\mathrm{X}$ : Nicousamide blocks the effects of advanced glycation end products on renal cells. Eur J Pharmacol 674: 455-459, 2012.

8. Sheng L, Chen H and Li Y: A HPLC method for determination of nicousamide in dog plasma and its application to pharmacokinetic studies. J Chromatogr B Analyt Technol Biomed Life Sci 854: 99-103, 2007.

9. Jakus V and Rietbrock N: Advanced glycation end-products and the progress of diabetic vascular complications. Physiol Res 53: 131-142, 2004.

10. Wendt TM, Tanji N, Guo J, et al: RAGE drives the development of glomerulosclerosis and implicates podocyte activation in the pathogenesis of diabetic nephropathy. Am J Pathol 162: $1123-1137,2003$

11. Tian D, Ling S, Chen G, et al: Hypertensive nephropathy treatment by heart-protecting musk pill: a study of anti-inflammatory therapy for target organ damage of hypertension. Int J Gen Med 4: 131-139, 2011.

12. Sun L, Ke Y, Zhu CY, et al: Inflammatory reaction versus endogenous peroxisome proliferator-activated receptors expression, re-exploring secondary organ complications of spontaneously hypertensive rats. Chin Med J (Engl) 121: 2305-2311, 2008.

13. Koshikawa S, Nishikimi T, Inaba C, Akimoto $\mathrm{K}$ and Matsuoka $\mathrm{H}$ Fasudil, a Rho-kinase inhibitor, reverses L-NAME exacerbated severe nephrosclerosis in spontaneously hypertensive rats. J Hypertens 26: 1837-1848, 2008.
14. Alfie J, Aparicio LS and Waisman GD: Current strategies to achieve further cardiac and renal protection through enhanced renin-angiotensin-aldosterone system inhibition. Rev Recent Clin Trials 6: 134-146, 2011.

15. Berl T: Review: renal protection by inhibition of the renin-angiotensin-aldosterone system. J Renin Angiotensin Aldosterone Syst 10: $1-8,2009$

16. Susic D, Frohlich ED, Kobori H, Shao W, Seth D and Navar LG Salt-induced renal injury in SHRs is mediated by AT1 receptor activation. J Hypertens 29: 716-723, 2011.

17. Varagic J, Ahmad S, Brosnihan KB, et al: Salt-induced renal injury in spontaneously hypertensive rats: effects of nebivolol. Am J Nephrol 32: 557-566, 2010.

18. Ehara T and Shigematsu H: Mast cells in the kidney. Nephrology (Carlton) 8: 130-138, 2003.

19. Jones C: Matrix degradation in renal disease. Nephrology (Carlton) 2: 13-23, 1996

20. Siragy HM: Angiotensin II compartmentalization within the kidney: effects of salt diet and blood pressure alterations. Curr Opin Nephrol Hypertens 15: 50-53, 2006. 